\title{
The MDM2 Oncoprotein Binds Specifically to RNA through Its RING Finger Domain
}

\author{
Brian Elenbaas,* Matthias Dobbelstein,*† Judith Roth,* \\ Thomas Shenk, ${ }^{*}$ and Arnold J. Levine* \\ *Department of Molecular Biology and ${ }^{\dagger}$ Howard Hughes Medical \\ Institute, Princeton University, Princeton, New Jersey, U.S.A.
}

\begin{abstract}
Background: The cellular $m d m 2$ gene has transforming activity when overexpressed and is amplified in a variety of human tumors. At least part of the transforming ability of the MDM2 protein is due to binding and inactivating the p53 tumor suppressor protein. Additionally, this protein forms a complex in vivo with the L5 ribosomal protein and its associated $5 \mathrm{~S}$ ribosomal RNA and may be part of a ribosomal complex.

Materials and Methods: A RNA homopolymer binding assay and a SELEX procedure have been used to characterize the RNA-binding activity of MDM2.

Results: The MDM2 protein binds efficiently to the homopolyribonucleotide poly $(\mathrm{G})$ but not to other homopolyribonucleotides. This binding is independent of the interaction of MDM2 with the L5 protein, which occurs through the central acidic domain of MDM2. An
\end{abstract}

RNA SELEX procedure was performed to identify specific RNA ligands that bind with high affinity to the human MDM2 (HDM2) protein. After 10 rounds of selection and amplification, a subset of RNA molecules that bound efficiently to HDM2 was isolated from a randomized pool. Sequencing of these selected ligands revealed that a small number of sequence motifs were selected. The specific RNA binding occurs through the RING finger domain of the protein. Furthermore, a single amino acid substitution in the RING finger domain, G446S, completely abolishes the specific RNA binding.

Conclusions: These observations, showing that MDM2 binds the $\mathrm{L} 5 / 5 \mathrm{~S}$ ribosomal ribonucleoprotein particle and can also bind to specific RNA sequences or structures, suggest a role for MDM2 in translational regulation in a cell.

\section{INTRODUCTION}

The $m d m 2$ oncogene (murine double minute 2) was originally discovered due to its gene amplification in a spontaneously transformed BALB/C $3 T 3$ cell line (1). Overexpression of $m d m 2$ is capable of immortalizing primary rat embryo fibroblasts and, in conjunction with an activated ras oncogene, of transforming these cells (2). The human $m d m 2$ gene ( $h d m 2$ ) has been found to be amplified in a significant number of soft tissue sarcomas, osteosarcomas, and gliomas (3-7). However, $m d m 2$ overexpression may result from mechanisms other than gene amplification. Translocation of $m d m 2$ with the immunoglobulin $C$ kappa gene results in $m d m 2$ overexpression in

Address correspondence and reprint requests to: Arnold J. Levine, Department of Molecular Biology, Princeton University, Princeton, NJ 08544-1014, U.S.A. the SP2 murine plasmacytoma cell line (8). In addition, $h d m 2$ is overexpressed at the RNA level without gene amplification in a large percentage of leukemias (9) and overexpressed due to enhanced translation in human choriocarcinoma cells (10).

The MDM2 protein is believed to be oncogenic by functioning as a negative regulator of the $\mathrm{p} 53$ tumor suppressor protein. MDM2 forms a tight complex with the N-terminal transactivation domain of $p 53$ and blocks the ability of p53 to transcriptionally activate gene expression (1113). This negative regulation of $\mathrm{p} 53$ has been shown to be essential in vivo from the analysis of $m d m 2$ null mice which exhibit embryonic lethality shortly after implantation. This phenotype is rescued in a $p 53$ null background, indicating that the negative regulation of $\mathrm{p} 53$ by MDM2 is essential during embryonic development $(14,15)$. 
In addition to binding and regulating p53, MDM2 has been shown to bind and regulate other proteins which may contribute to the oncogenic potential of the protein. MDM2 has been shown to bind the retinoblastoma protein $\mathrm{pRB}$ and relieve its negative regulation of the transcriptional activator E2F1 (16). MDM2 has also been reported to bind directly to the E2F1/DP1 transcription factor and stimulate its transcriptional activity (17). Additionally, MDM2 has been shown to form a tight complex in vivo with the $\mathrm{L} 5$ ribosomal protein, which in turn is associated with the 5S ribosomal RNA (18). However, the function of this complex is currently unclear.

The binding domain for $\mathrm{p} 53$ and E2Fl has been mapped to the $\mathrm{N}$-terminal domain of MDM2, a domain which is sufficient for the negative regulation of p53 $(11,17,19)$. However, the MDM2 protein contains several additional highly conserved domains at the amino acid sequence level including a central acidic domain adjacent to a $\mathrm{C}_{4}$ zinc finger domain, and a $\mathrm{C}$-terminal RING finger domain (20). The RING finger is a $\mathrm{C}_{3} \mathrm{HC}_{4}$ domain that coordinates two molecules of zinc and folds as a single domain (21-23). A domain of analogous structure is found in over 40 proteins and has been speculated to function as either a nucleic acid binding domain or a protein-protein interaction domain (24).

This study investigates the biochemical functions of the acidic, zinc finger, and RING finger domains of MDM2. First, MDM2 was shown to efficiently interact with the poly(G) homopolymer of RNA. The interaction of MDM2 with the L5 ribosomal protein occurred through the central acidic domain of MDM2 and this domain was not required for poly $(G)$ binding. Specific RNA molecules that could bind to the protein were identified by in vitro selection from a randomized RNA pool, using a SELEX (systematic evolution of ligands by exponential enrichment) procedure. The specific RNA binding was shown to occur through the RING finger domain of MDM2 and was completely abolished by a single point mutation in the RING finger.

\section{MATERIALS AND METHODS}

\section{Baculovirus Protein Expression and Purification}

MDM2, HDM2, and HDM2 deletion mutants (11) were expressed by cloning the cDNAs into
PVL1393 and cotransfection with baculogold viral DNA into Sf9 insect cells (Pharmingen, San Diego, CA, U.S.A.). HA epitope-tagged proteins (HA HDM2 and HA L5) were constructed by cloning oligonucleotides encoding the nine amino acid HA epitope into PVL 1393 so that $\mathrm{N}$-terminal fusion proteins could be generated.

MDM2 for use in RNA homopolymer binding assays was partially purified by single-step immunoaffinity purification. Sf9 cells were infected with a MDM2 baculovirus for $48 \mathrm{hr}$, harvested, and lysed for $30 \mathrm{~min}$ on ice with lysis buffer: $50 \mathrm{mM}$ Tris (pH 7.5), $150 \mathrm{mM} \mathrm{NaCl}, 0.5 \%$ Nonidet P-40, 1 mM DTT, and protease inhibitors (0.2 mM PMSF, $1 \mu \mathrm{M}$ pepstatin A, and $1 \mu \mathrm{M}$ E-64). The lysate was centrifuged at $40,000 \times g$ for $20 \mathrm{~min}$ at $4^{\circ} \mathrm{C}$, and the supernatant was peristaltically pumped through a Sepharose CL-4B pre-column followed by a protein A-Sepharose 2A10 monoclonal antibody (mAb) immunoaffinity column at a flow rate of $0.4 \mathrm{ml} / \mathrm{min}$. The 2Al0 immunoaffinity column was constructed by coupling of antibody to protein A with dimethyl pimelimidate as described (25). The lysate was passed over the column three times and the immunoaffinity column washed with $50 \mathrm{col}-$ umn volumes of SNNT buffer ( $5 \%$ sucrose, $1 \%$ Nonidet P-40, $0.5 \mathrm{M} \mathrm{NaCl}, 50 \mathrm{mM}$ Tris [pH 7.5], $1 \mathrm{mM}$ DTT, and protease inhibitors) followed by 50 volumes of RIPA buffer ( $50 \mathrm{mM}$ Tris [pH 7.5], $150 \mathrm{mM} \mathrm{NaCl}, 1 \%$ Triton $\mathrm{X}-100,1 \%$ sodium deoxycholate, $1 \mathrm{mM}$ DTT and protease inhibitors) and finally 50 volumes of lysis buffer.

MDM2 was eluted from the column by first washing the column in $10 \mathrm{mM}$ sodium phosphate buffer ( $\mathrm{pH}$ 6.8) and eluting the MDM2 with $100 \mathrm{mM}$ Glycine $(\mathrm{pH} 2.5)$ into collection tubes containing $1 \mathrm{M}$ Tris $(\mathrm{pH} 8)$. Fractions containing MDM2 were dialyzed in Final Storage Buffer (20 mM Tris [pH 7.5], $150 \mathrm{mM} \mathrm{NaCl}$, $0.1 \%$ Nonidet P-40, 20\% Glycerol, 1 mM DTT and protease inhibitors) and concentrated by ultrafiltration in a centricon-30 (Amicon, Beverly, MA, U.S.A.).

HA HDM2 protein used in the SELEX procedure was purified as described with a $12 \mathrm{CA} 5 \mathrm{mAb}$ immunoaffinity column except that the protein was eluted with the nine amino acid peptide corresponding to the HA epitope (n-YPYDVPDYA-c). After the column was washed in lysis buffer, the resin was transferred to a $15-\mathrm{ml}$ conical tube and eluted with four volumes of lysis buffer containing $1 \mathrm{mg} / \mathrm{ml} \mathrm{HA}$ peptide overnight at $4^{\circ} \mathrm{C}$ with rocking. The eluate was dialyzed against Final 
Storage Buffer using a membrane with a $12-\mathrm{kD}$ MWCO and concentrated in a centricon- 30 .

\section{RNA-Binding Assays}

Binding of purified MDM2 to the different nucleic acid columns in Fig. 1 was done essentially as described (26) by incubating $0.5 \mu \mathrm{g}$ MDM2 with the columns for $1 \mathrm{hr}$ at $4^{\circ} \mathrm{C}$ with rocking in a total volume of $300 \mu \mathrm{l}$ RNA-binding buffer (20 $\mathrm{mM}$ Tris [pH 7.5], $150 \mathrm{mM} \mathrm{NaCl}, 5 \mathrm{mM} \mathrm{MgCl}_{2}$, $0.1 \%$ Nonidet P-40, $50 \mu \mathrm{M} \mathrm{ZnCl}_{2}, 2 \%$ Glycerol, 1 mM DTT). MDM2 was incubated with about 20 $\mu \mathrm{g}$ nucleic acid on the following columns after each was washed in RNA-binding buffer: ds calf thymus DNA cellulose (Sigma, Chemical Co., St. Louis, MO, U.S.A.), denatured calf thymus DNA agarose (Pharmacia, Piscataway, NJ, U.S.A.), and the four RNA homopolymer columns (Sigma). Columns were washed with $3 \mathrm{ml}$ of RNA-binding buffer and boiled in SDS sample buffer, and the protein was separated by SDS-PAGE. The gel was transferred to nitrocellulose for western blotting, followed by detection with the 2A10 $\mathrm{mAb}$ and ECL chemiluminescence (Amersham, Arlington Heights, IL, U.S.A.).

The immunoprecipitation/RNA-binding assay (Figs. 2, 4, 6, and 7) was done by immunoprecipitating about $1 \mathrm{mg}$ Sf9 lysate with $200 \mu \mathrm{l}$ of mAb supernatant, $30 \mu \mathrm{l} 50 \%$ protein A-Sepharose beads, lysate, and lysis buffer to a final volume of $450 \mu \mathrm{l}$ at $4^{\circ} \mathrm{C}$ for $2 \mathrm{hr}$. Immunoprecipitates were washed with $2 \times 1 \mathrm{ml}$ of SNNT buffer and $1 \mathrm{ml}$ of RNA-binding buffer. RNA-binding buffer $(300 \mu \mathrm{l})$ containing $0.1 \mu \mathrm{g}$ of ${ }^{32} \mathrm{P}$-labeled RNA and $20 \mu \mathrm{g}$ each of unlabeled poly(U) RNA and Torula yeast RNA (Sigma) as nonspecific competitors. Poly $(G)$ RNA was end-labeled with T4 polynucleotide kinase and $\left[\gamma^{32} \mathrm{P}\right]$ ATP. The RNA from the SELEX rounds was generated by in vitro transcription with T7 RNA polymerase (Ambion, Austin, TX, U.S.A.) in the presence of $\left[\alpha-{ }^{32} \mathrm{P}\right]$ UTP. RNA-binding reactions were carried out at RT for $1 \mathrm{hr}$ with rocking followed by $3 \times$ $1 \mathrm{ml}$ washes in RNA-binding buffer, after which the beads were resuspended in $80 \mu \mathrm{l}$ of buffer, dissolved in scintillation fluid, and counted.

\section{Immunoprecipitation of MDM2 and HA L5}

Sf9 cells were co-infected with baculoviruses for expression of HA L5 protein along with a virus for expression of MDM2, HDM2, or a deletion mutant of HDM2 for $44 \mathrm{hr}$ in $10-\mathrm{cm}$ plates. Sf9 cells were then transferred to a $15-\mathrm{ml}$ conical tube, pelleted by brief centrifugation, and resuspended in $4 \mathrm{ml}$ of methionine-deficient insect cell media (Ex-cell 401 deficient, JRH Biosciences, Lenexa, KS, U.S.A.). Cells were then returned to $10-\mathrm{cm}$ plates followed by the addition of $75 \mu \mathrm{Ci} / \mathrm{ml}\left[{ }^{35} \mathrm{~S}\right]$ methionine Express (DuPont, Wilmington, DE, U.S.A.) and incubation for $4 \mathrm{hr}$. Cells were washed in PBS and lysed in lysis buffer. The lysate was centrifuged and subjected to immunoprecipitation as described above. Immunoprecipitates were washed with $2 \times 1 \mathrm{ml}$ of SNNT buffer and $1 \mathrm{ml}$ of RIPA buffer, boiled in sample buffer, and separated by SDSPAGE. Gels were subjected to fluorography, dried, and exposed to film as described (11).

\section{SELEX Procedure}

The randomized RNA library was generated by in vitro transcription of a 77-base template oligonucleotide containing a central 30 nucleotide randomized region. The sequence of the template oligonucleotide and the primers for in vitro transcription, reverse transcription, and polymerase chain reaction (PCR), along with the conditions for these reactions was as previously described $(27,28)$. Baculovirus-produced, HAtagged HDM2 was purified by $12 \mathrm{CA} 5$ immunoaffinity chromatography and peptide eluted as described above. Purified HA HDM2 protein was coupled to $12 \mathrm{CA} 5$ antibody that was immobilized on protein A-Sepharose beads as described above. RNA pools were incubated with the immobilized HDM2 in SELEX buffer $(20 \mathrm{mM}$ Tris [pH 7.5], $250 \mathrm{mM} \mathrm{NaCl}, 0.1 \mathrm{mM}$ EDTA, $1 \mathrm{mM}$ $\mathrm{MgCl}_{2}, 0.05 \%$ Nonidet P-40, $1 \mathrm{mM}$ DTT) for $1 \mathrm{hr}$. After five washes with the same buffer, the complexes of HDM2 and RNA were eluted with the HA peptide ( $1 \mathrm{mg} / \mathrm{ml}$ in SELEX buffer) for 30 min. The RNA was purified by phenol extraction and ethanol precipitation, followed by reverse transcription and PCR amplification as described (27).

\section{GST HDM2 RING Finger Expression}

The fusion protein was constructed by PCR amplification of the RING finger domain with the oligonucleotide 5'-CGGAATTCCAGCCATCAAC TTCTAGTAGC- $3^{\prime}$ and the T7 primer (5'-GTAAT ACGACTCACTATAGGGC-3') from the pHDM $\Delta 1-152$ plasmid which has this fragment of $h d m 2$ cloned into the EcoRI site of pBluescript KS- (11). The fragment was digested with EcoRI and cloned 
into pGEX-4T-1 (Pharmacia). Upon induction with $1 \mathrm{mM}$ IPTG for $2 \mathrm{hr}$ in BL21 cells, this construct produced a fusion protein containing amino acids 396-491 of HDM2. Purification of the GST and GST HDM2 RING finger proteins was performed by lysing induced cells in lysis buffer with sonication, centrifugation, and isolation with glutathione agarose beads (Pharmingen) or protein A-Sepharose and mAbs as described above.

\section{RESULTS}

\section{MDM2 Binds Preferentially to the Homopolyribonucleotide poly(G)}

In order to investigate functions of MDM2 in addition to the regulation of $\mathrm{p} 53$, experiments were carried out to characterize the protein's zinc finger domains. Zinc fingers have often been found to mediate interactions with nucleic acids. In addition, it was noticed that MDM2 binds to a heparin-sepharose column which is characteristic of many nucleic acid binding proteins. We made use of a semispecific assay that has been used extensively to study the RNA-binding properties of the hnRNP and other proteins (29-31). In this assay, a protein is tested for its ability to interact with DNA or RNA by using calf thymus DNA or RNA homopolymers immobilized on agarose beads.

Baculovirus-produced, purified MDM2 protein was incubated with six different columns containing ds DNA or ss DNA from calf thymus, or each of the four natural homopolyribonucleotides. Bound MDM2 protein was visualized by SDS-PAGE and western blotting with a monoclonal antibody (mAb) against MDM2 (Fig. 1). MDM2 bound most efficiently $(\sim 5 \%$ of input protein) to the poly $(G)$ RNA column (Fig. 1, Lane 5$)$. Less efficient binding ( $\sim 0.5 \%$ of input protein) could be detected to ds DNA (Fig. 1, Lane 1) and poly(U) RNA (Fig. 1, Lane 6). This specificity for poly $(G)$ RNA is very similar to that of several recently characterized RNA-binding proteins $(26,32)$.

A second RNA-binding assay was performed by first immunoprecipitating MDM2 or HDM2 from baculovirus-infected Sf9 insect cell lysates with various mAbs. This was followed by incubation of the immobilized protein with radiolabeled poly(G) RNA, washing, and counting the beads in a scintillation counter. As shown in Fig. 2A, both MDM2 and HDM2 bound poly(G) RNA in this assay when immunoprecipitations

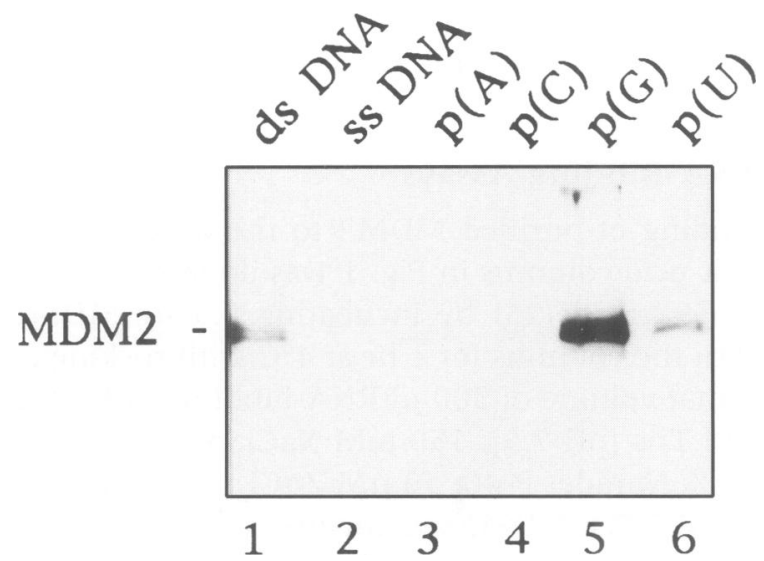

FIG. 1. Western analysis of baculovirus-produced, purified MDM2 protein bound to six columns containing ds DNA, ssDNA, or each of the four homopolyribonucleotides as indicated (Lanes 3-6)

were performed from insect cell lysates overexpressing MDM2 or HDM2 with mAbs to MDM2 (2A10, 4B11, and 4B2). These mAbs each recognize distinct epitopes in different regions of MDM2: 4B2, an N-terminal epitope (amino acids 1-58); 2A10, a central epitope (amino acids 295339); and 4B11, a C-terminal epitope (amino acids 396-444). Low, background levels of RNA binding were seen with immunoprecipitations from uninfected or wild-type virus infected Sf9 lysates or with negative control antibodies (419 and 421 ). The total cpm of radiolabeled RNA was always 5- to 20-fold above the background levels (i.e., immunoprecipitates of uninfected cells with the same MDM2 mAbs). The poly(G) RNA binding efficiently decreases with increasing $\mathrm{NaCl}$ concentrations in the RNA-binding buffer (Fig. 2B), indicating that ionic interactions contribute to the association. The poly $(\mathrm{G})$ RNA binding is not competed by excess yeast Torula RNA or poly (dI-dC) (Fig. 2B). Thus, both MDM2 and HDM2 bind poly(G) RNA efficiently and specifically.

\section{The RNA Binding Is Independent of the Interaction of MDM2 with the L5 Ribosomal Protein}

MDM2 has previously been shown to form a complex in vivo with the L5 protein/5S rRNA ribonucleoprotein particle, a component of the large ribosomal subunit (18). Furthermore, a complex of p53-MDM2-L5-5S RNA was shown to exist since the 5S RNA could be detected in an 
A.

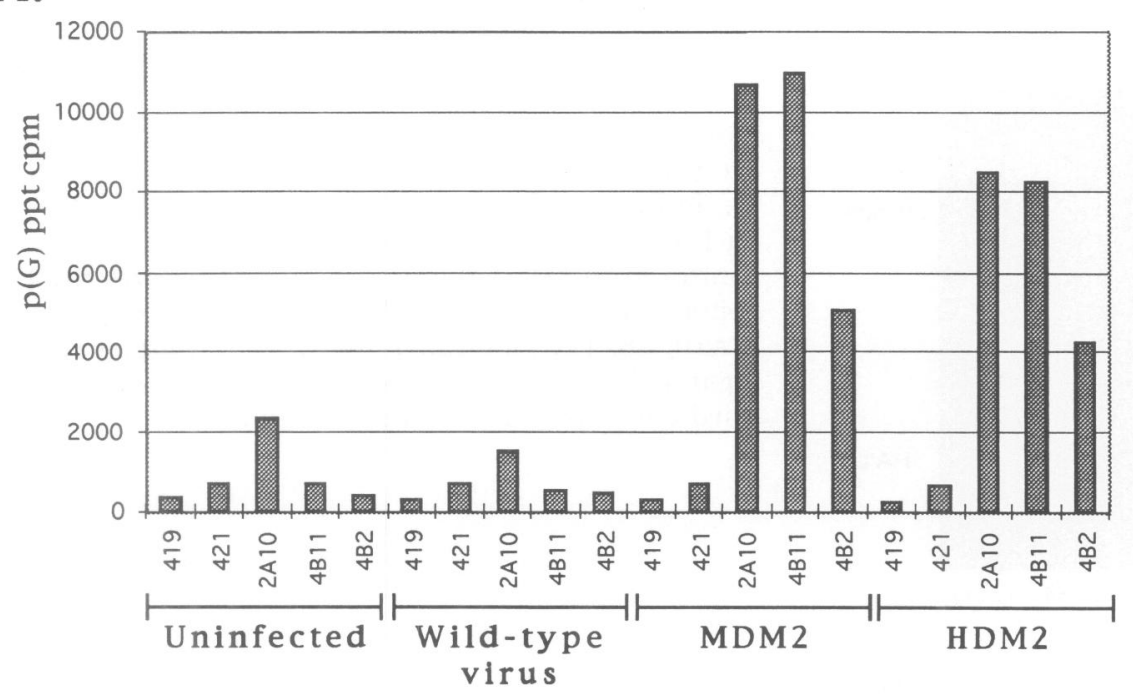

B.

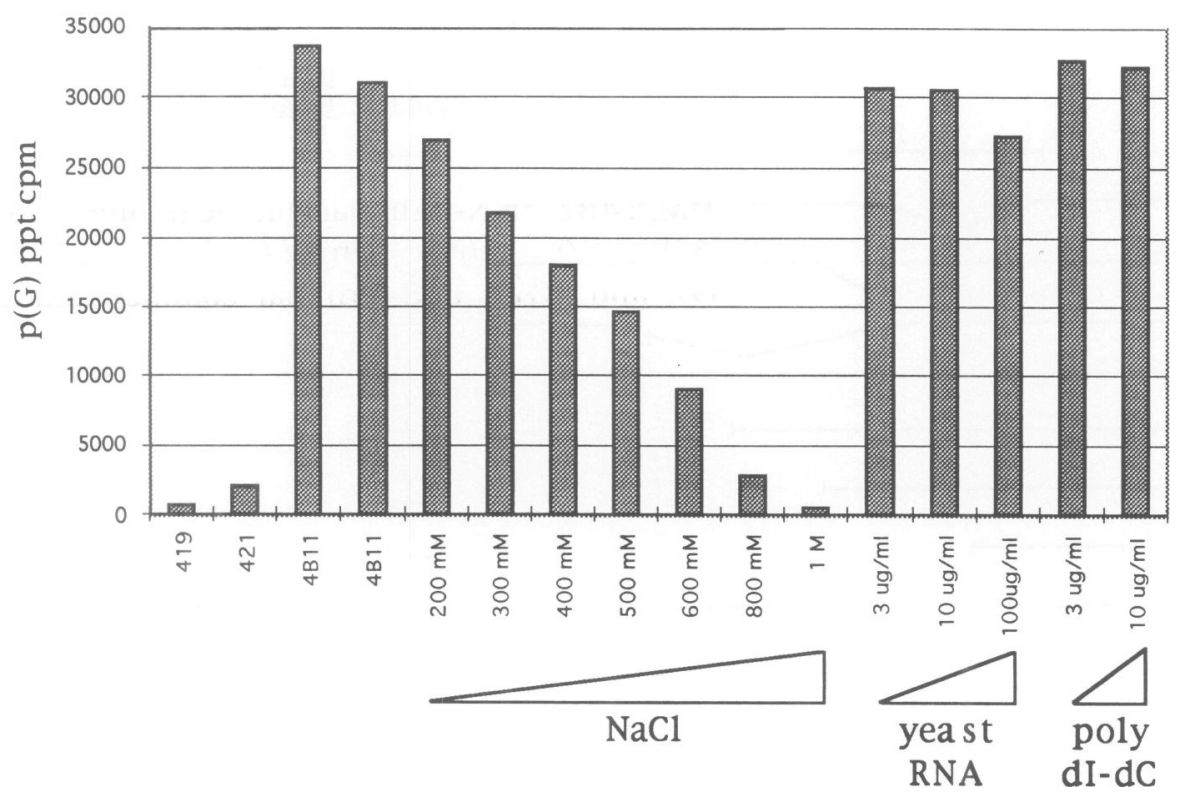

FIG. 2. MDM2 and HDM2 bind poly(G) RNA when immunoprecipitated from Sf9 insect cell lysates overexpressing these proteins $(A)$ and binding of MDM2 to poly(G) RNA decreases with increasing $\mathrm{NaCl}$ concentration and is not competed with excess yeast RNA or poly(dIdC) (B)

(A) Immunoprecipitations were performed from Sf9 lysates that were uninfected, infected with a wild-type AcMNPV baculovirus, or recombinant baculoviruses which overexpress MDM2 or HDM2 as indicated. Each lysate was immunoprecipitated with 2 negative control mAbs $(419,421)$ and 3 mAbs specific for MDM2 and HDM2 (2A10, 4B 11 and 4B2). The beads were washed and incubated with $\left[{ }^{32} \mathrm{P}\right]$ end-labeled poly(G) RNA in an RNAbinding buffer. The beads were then washed and counted in a scintillation counter. (B) Sf9 lysate overexpressing MDM2 was immunoprecipitated with 419, 421 (Columns 1 and 2) or 4B11 (Columns 3-16). RNA-binding reactions were performed with increasing $\mathrm{NaCl}$ concentration, with excess yeast Torula RNA, and excess poly $(\mathrm{dI}-\mathrm{dC})$ as indicated. immunoprecipitation of $\mathrm{p} 53$ from cells under conditions when large amounts of p53-MDM2 complex are detectable (18). Since MDM2 forms this complex with the L5 protein, a known RNAbinding protein, it became necessary to determine whether the poly $(G)$ RNA binding was direct or mediated through the $\mathrm{L} 5$ protein. In other words, it remained possible that the MDM2 protein, when overexpressed in Sf9 insect cells, bound to the endogenous insect L5 which was capable of binding poly $(\mathrm{G})$.

In order to determine whether or not the
RNA binding was mediated through the L5 protein, the domains of MDM2 responsible for the interaction with L5 and poly $(G)$ were determined. A panel of deletion mutants of HDM2 was generated in baculovirus expression vectors along with a hemagglutinin (HA) epitope-tagged mouse L5 protein. Sf 9 cells were then co-infected with baculoviruses for expression of each of the HDM2 deletion mutants and HA L5, labeled with $\left[{ }^{35} \mathrm{~S}\right]$ methionine, and immunoprecipitated with a negative control antibody (419), the HA antibody (12CA5), and antibodies to HDM2 (2A10 


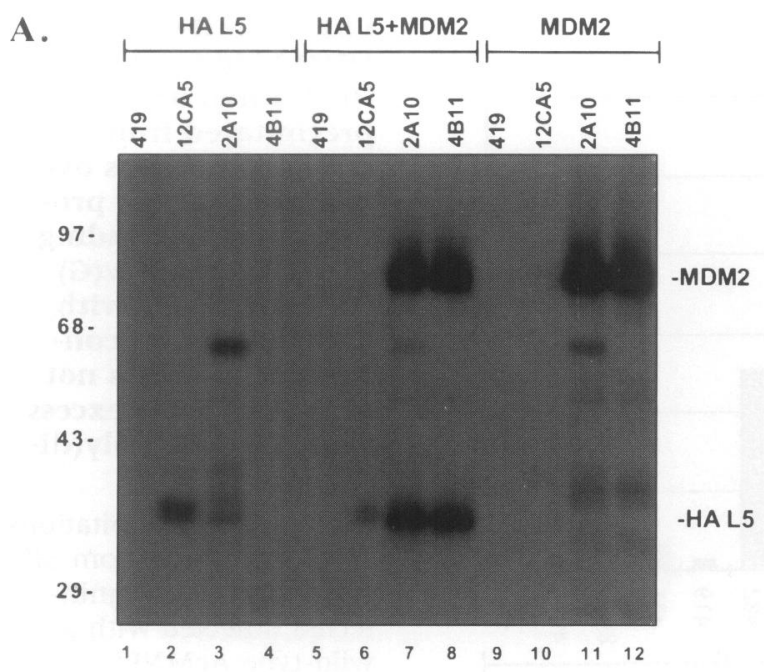

FIG. 3. MDM2 forms a complex with HA-tagged L5 protein when co-expressed in Sf9 cells (A); summary of mapping data of nine deletion $\mathrm{mu}-$ tants of HDM2 for binding to the L5 protein and $\operatorname{poly}(G)$ RNA (B)

(A) Sf9 cells were infected with baculoviruses for expression of either HA L5, HA L5 + MDM2, or MDM2 for $44 \mathrm{hr}$ and labeled with $\left.{ }^{35} \mathrm{~S}\right]$ methionine for $4 \mathrm{hr}$. Each lysate was immunoprecipitated with a negative control mAb (419), the mAb which recognizes the HA epitope (12CA5), or $2 \mathrm{mAbs}$ which recognize MDM2 (2A10, 4B 11) as indicated. Immunoprecipitates were separated on an $8 \%$ SDS polyacrylamide gel which was dried and exposed to autoradiography.

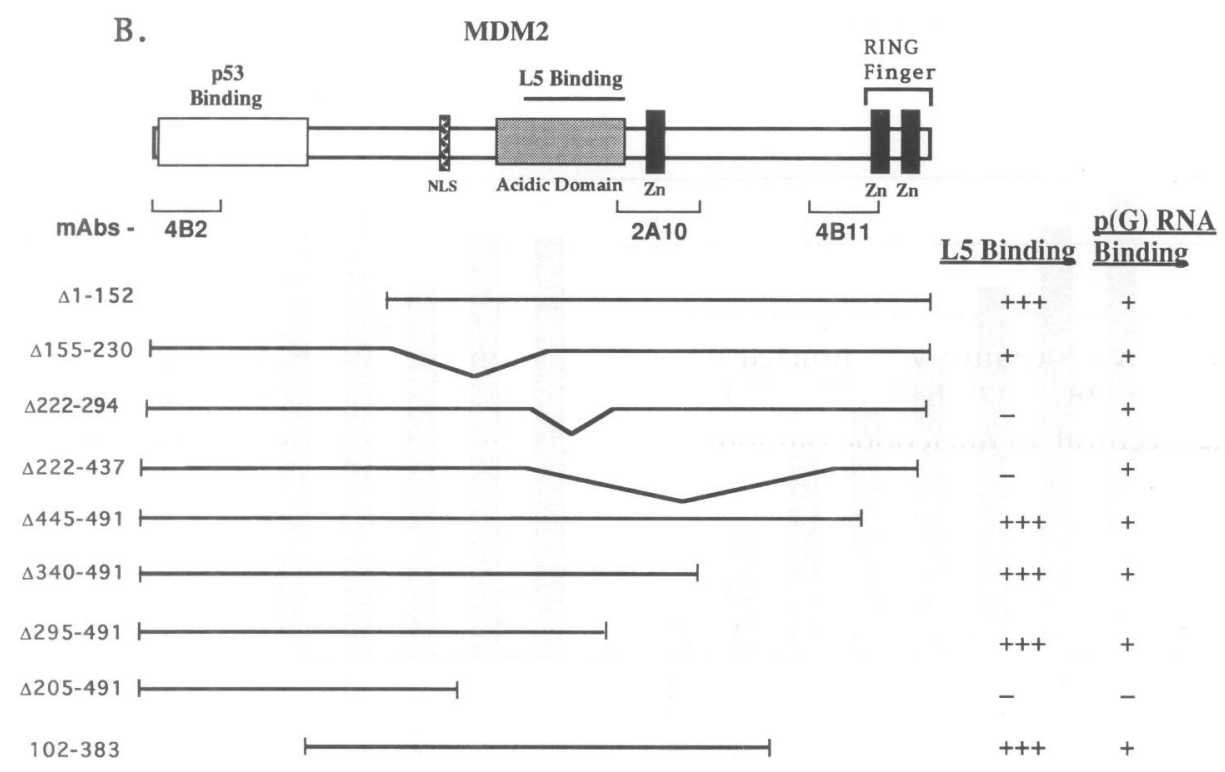

and 4B 1 1). An example of this assay is shown in Fig. 3A where the complex between MDM2 and HA L5 was easily detectable when Sf9 cells were co-infected and immunoprecipitated with MDM2 mAbs (Fig. 3A, Lanes 7 and 8) even in high salt $(500 \mathrm{mM} \mathrm{NaCl})$ washes. Immunoprecipitation with the $12 \mathrm{CA} 5 \mathrm{mAb}$ failed to detect the MDM2-L5 complex, possibly because the HA tag on L5 was masked by MDM2 complexed with it or the 12CA5 mAb was not capable of quantitatively precipitating the HA L5 protein. HDM2 bound as efficiently as MDM2 to L5 (data not shown).

Next, the ability of HDM2 deletion mutants to bind L5 was tested. The results are summarized in Fig. 3B. Deletion of the $\mathrm{N}$-terminal do- main $(\Delta 1-152)$ or the $C$ terminus $(\Delta 295-491)$ does not affect L5 binding, but deletion of the central domain $(\Delta 222-294, \Delta 205-491)$ completely eliminates binding. The $\Delta 155-230$ deletion partially interferes with the interaction with L5 indicating that these amino acids contribute to L5 binding. Furthermore, the central 102-383 fragment of HDM2 was shown to bind L5 demonstrating that the central acidic domain is sufficient for binding the L5 protein. Thus, the central acidic domain of HDM2 (amino acids 153-294) is responsible for the interaction with L5.

In order to map the region responsible for the poly $(G)$ RNA binding, each of the HDM2 deletion mutants was tested in the RNA-binding 
assay used in Fig. 2, and a summary of the results is shown in Fig. 3B. Two mutants, $\Delta 222-294$ and $\Delta 222-437$, which fail to bind L5, are able to bind poly(G) RNA, indicating that the RNA binding is not mediated through the L5 protein. Surprisingly, only the $\mathrm{N}$-terminal fragment of HDM2 consisting of amino acids 1-204 failed to recognize poly $(G)$ RNA. Differences in the relative affinity of the remaining mutants for poly $(G)$ was not observed. When a C-terminal portion of the protein is attached to the $\mathrm{N}$-terminal fragment, RNA binding was restored. These mapping data indicate that there are two regions on HDM2 that are capable of binding poly(G) RNA. One of them resides in the central region because $\Delta 295-491$ binds but not $\Delta 205-491$. A second RNA-binding region is located at the $C$ terminus because deletions of the central domain $(\Delta 222-$ 294 and $\Delta 222-437)$ retain the ability to recognize RNA.

\section{HDM2 Binds Specifically to RNA}

In order to determine whether HDM2 is capable of binding to a specific RNA sequence or structural motif, a SELEX procedure was utilized as described previously (28). A 77-base oligonucleotide containing a central 30 nucleotide randomized region was transcribed using T7 RNA polymerase to create a randomized pool of RNA molecules. This pool, containing approximately $10^{12}$ different RNA molecules, was incubated with baculovirus-produced, purified HA-tagged HDM2 protein, and protein A-Sepharose beads coupled with the 12CA5 mAb. The beads were washed and the HDM2-RNA complexes eluted from the beads with the nine amino acid HA peptide. The RNA was subjected to reverse transcription followed by PCR amplification, and the DNA used to transcribe another pool of RNA. This process was repeated 10 times in order to selectively enrich for a population of RNA molecules that bound with high affinity to the HDM2 protein. A pool of RNA molecules (Round 10) was isolated that bound efficiently to HDM2 while no binding was detected with the initial pool (Round 0). By using the same RNA-binding assay as in Fig. 2, RNA binding was only detected with Round 10 RNA when immunoprecipitations were performed from insect cell lysates expressing HDM2 with mAbs to HDM2 (4B2 and 2A10) (Fig. 4).

The PCR-amplified 77-nt DNA that was used as the template to transcribe the Round 10 RNA was cloned into the pUC19 plasmid. Sequencing

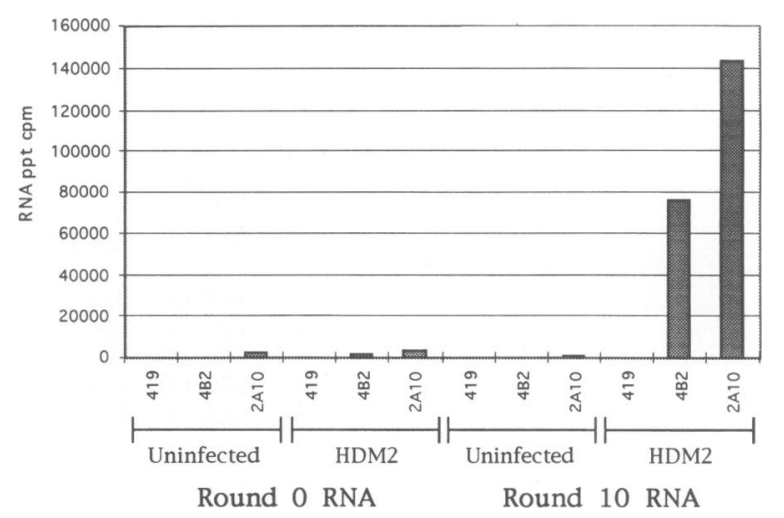

FIG. 4. HDM2 binds specifically to Round 10 RNA generated from the SELEX procedure

The RNA-binding assay was performed as in Fig. 2 with two pools of radiolabeled RNA from the SELEX procedure; either the initial pool (Round 0) or the final pool (Round 10). Uninfected and HDM2 expressing Sf9 lysates were immunoprecipitated with the 419 negative control $\mathrm{mAb}$ and the HDM2 mAbs (4B2, 2A10) as indicated.

of 36 individual clones revealed that one predominant RNA molecule had been selected, representing 16 of 36 clones. This molecule was designated clone $A$. Several other clones selected (designated I, B, E, and L) differed from clone A in their central 30 nucleotides by only 1 or 2 nucleotides. Another clone was found in 2/36 clones and differed at 5 nucleotides (clone $\mathrm{K}$ ) (Fig. 5A). Together, clone A and similar clones accounted for $28 / 36(78 \%)$ of the clones, and the remaining eight clones each appeared once and were unrelated to clone A. Importantly, when individual clones were transcribed and tested for binding, HDM2 bound to the clone $\mathrm{A}$ and $\mathrm{K}$ RNAs, but not to RNA from two of the unrelated clones, $\mathrm{D}$ and $\mathrm{F}$ (data not shown).

The in vitro-selected RNAs were folded according to the method described by Zuker (33), and the predicted structure of clone A RNA is shown in Fig. 5B. The clones similar to clone $A$ in Fig. 5A are all predicted to form this structure. The nucleotides that differ from clone $A$ in the similar clones are indicated. This structure consists of a 5' stem-loop and a $3^{\prime}$ region containing a large single-stranded region of $13 \mathrm{nt}$ and a loop of $7 \mathrm{nt}$. The 30-nt randomized region forms a stem with the $3^{\prime}$ primer-binding sequences. Stems that contain primer-binding sequences commonly occur in RNA aptamers and are often found to buttress functional domains (34). The incorporation of defined sequences into struc- 


\section{HDM2 RNA Consensus Sequences}
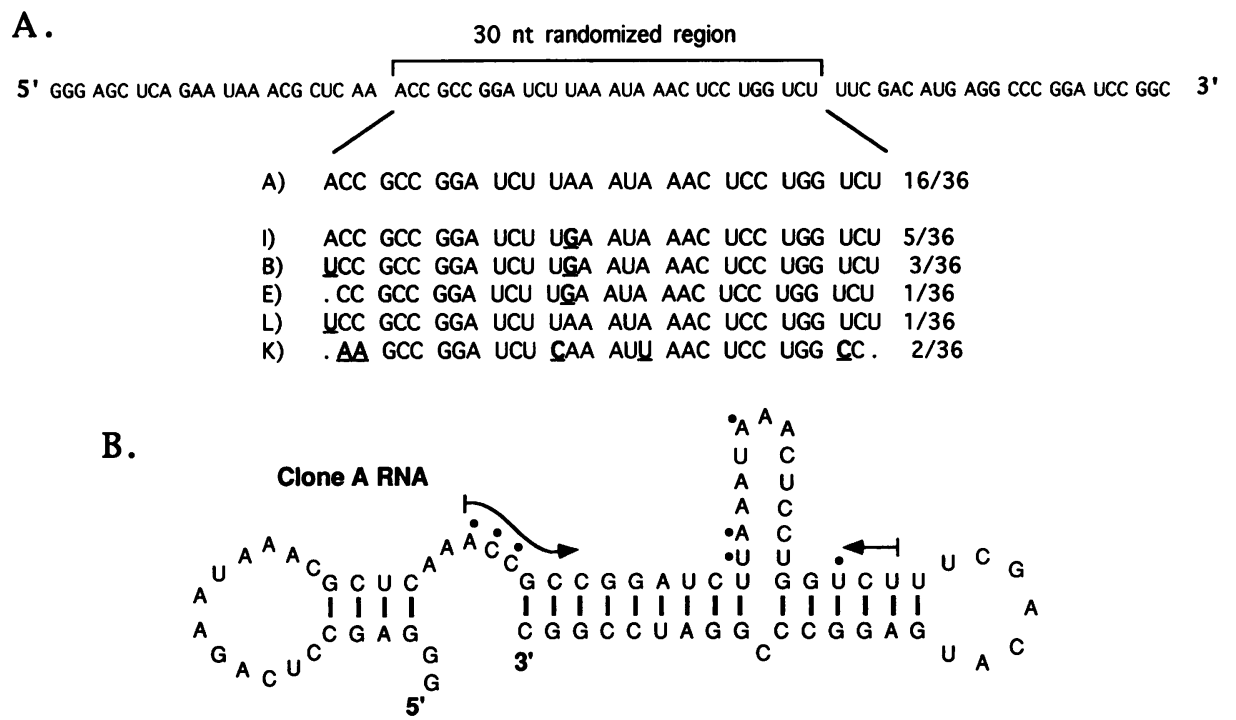

FIG. 5. Sequence of the clone $A 77$ nt RNA isolated from the SELEX procedure containing the central 30 nt randomized region flanked by the $5^{\prime}$ and $3^{\prime}$ defined sequences used for reverse transcription, in vitro transcription, and PCR amplification of the pool (A) and predicted secondary structure of clone A RNA (B)

(A) The PCR-amplified DNA used to transcribe the Round 10 RNA was cloned into pUC19 for DNA sequencing. Thirty-six clones were sequenced and the most common sequence, designated $A$, along with related sequences (I, B, E, L, and K) are shown followed by the number of occurrences of each. Bold, underlined nucleotides in (I, B, E, L, and K) indicate differences with the A sequence. Eight unrelated sequences that occurred once are not shown. (B) Arrows, the 30-nt sequence from the previously randomized region. The nucleotides in clones I, B, E, $\mathrm{L}$, and $\mathrm{K}$ that differ from clone $\mathrm{A}$ are indicated by an adjacent point.

tural supports is expected since only one rather than both strands of the stem must be derived from randomized sequence regions during the course of selection.

Two observations support the notion that this structure is important for recognition by HDM2. First, the RNA molecules unrelated in sequence to clone $\mathrm{A}$ are not predicted to form this structure and, as far as examined (D and F), did not detectably bind to HDM2. Second, an RNA molecule was generated that contains 13 additional nucleotides derived from a polylinker sequence on the 3' end of the clone A RNA. This 90-nt RNA is predicted to form an entirely different structure from clone $\mathrm{A}$ and fails to bind HDM2 (data not shown). Thus, it appears that the formation of this structure is critical for recognition by HDM2.

\section{The RING Finger Domain of HDM2 Is Sufficient for Specific RNA Binding}

The domain on HDM2 responsible for the specific RNA binding was first mapped using three of the deletion mutants shown in Fig. $3 \mathrm{~B}$ and the Round 10 RNA (Fig. 6A). Immunoprecipitation of the full-length HDM2 or an N-terminal deletion ( $\Delta 1-152)$ with HDM2 mAbs followed by incubation with Round 10 RNA revealed that each of these proteins bound efficiently to RNA. The difference in the RNA-binding ability of fulllength HDM2 and $\Delta 1-152$ as seen with $2 A 10$ is likely due to the lower expression levels of the two proteins in Sf9 cells (data not shown). Significantly, deletion of the RING finger domain $(\Delta 445-491)$ or a larger portion of the $C$ terminus $(\Delta 340-491)$ resulted in a complete loss of RNAbinding ability.

To determine whether the RING finger was sufficient for RNA binding and to rule out the possibility that the RNA binding was mediated through an insect cell protein that bound to the RING finger, a fusion protein of glutathione Stransferase (GST) and the HDM2 RING finger domain was expressed in Escherichia coli and tested for RNA binding. The GST RING finger fusion protein was composed of the $96 \mathrm{C}$-terminal amino acids of HDM2 (396-491). This fusion 


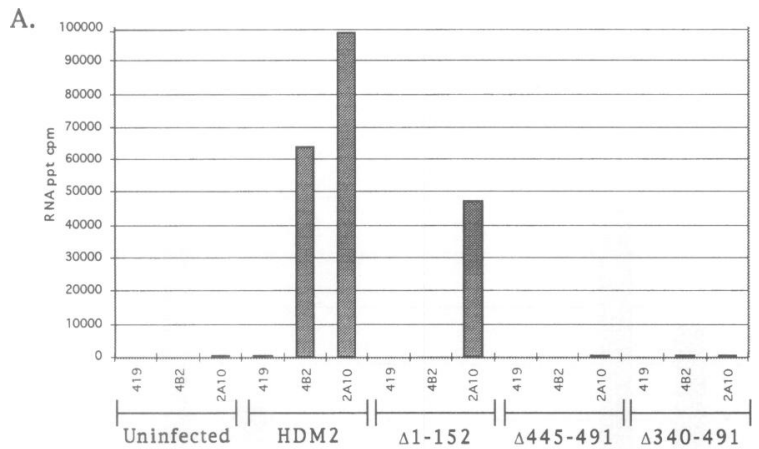

B.

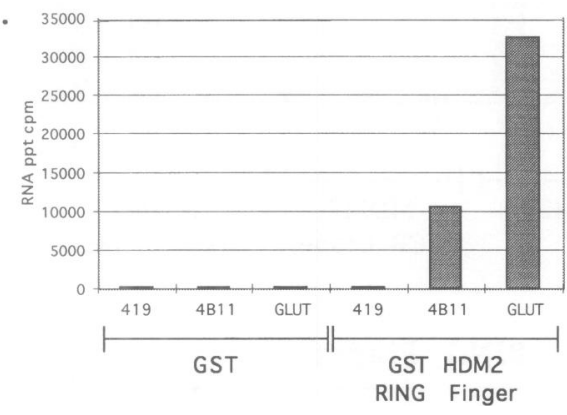

FIG. 6. The RING finger domain of HDM2 is sufficient for specific RNA binding

(A) Three HDM2 deletion mutants (Fig. 3B) were tested for their ability to bind Round 10 RNA as in Fig. 4 with the negative control mAb 419 or the HDM 2 mAbs $4 \mathrm{~B} 2$ and $2 \mathrm{Al} 0$ as indicated. Immunoprecipitation of the $\Delta 1-152$ mutant with $4 \mathrm{~B} 2$ failed to show RNA binding as expected because 4B2 maps to the $\mathrm{N}$ terminus and fails to bind this protein. (B) The GST protein and a GST HDM2 RING finger fusion protein were tested for their ability to bind Round 10 RNA using the same RNA-binding assay. Immunoprecipitations were performed from $E$. coli lysates overexpressing GST or the GST HDM2 RING finger protein with the $419 \mathrm{mAb}$ and the HDM2 mAb 4B 11 or glutathione beads (GLUT) as indicated.

protein, but not GST alone, is sufficient for binding the Round $10 \mathrm{RNA}$ as shown by selection with either the 4B11 HDM2 mAb or glutathione beads (Fig. 6B). The difference in RNA binding between $4 \mathrm{Bll}$ and the glutathione beads is possibly due to the fact that the $4 \mathrm{Bll}$ mAb recognizes an epitope adjacent to the RING finger (amino acids 396-444) and might therefore partially interfere with the ability of the protein to bind RNA. In fact, this monoclonal antibody always detected less RNA binding to full-length HDM2 than N-terminal HDM2 antibodies (data not shown). The GST RING finger protein was incubated with $5 \mathrm{~S}$ rRNA, but all attempts have failed to detect any binding (data not shown).
The fact that an HDM2 protein domain from two different sources, baculovirus-infected cells and $E$. coli, bound specifically to Round 10 RNA strongly argues that the RNA binding is direct.

\section{A G446S Point Mutation in the MDM2 RING Finger Completely Abolishes Specific RNA Binding}

There is a high degree of amino acid conservation between HDM 2 and MDM 2 across the RING finger region (only four amino acid differences across a 68-amino acid region shown in Fig. 7B). Because of this, MDM2 (the murine protein) was tested to determine if it was capable of binding Round 10 RNA. MDM2 produced from the $\mathrm{P}_{2}$ cDNA clone (35) bound Round 10 RNA as well as full-length HDM2 (Fig. 7A). In contrast, another $m d m 2$ clone which was originally generated by reverse transcription and PCR failed to bind specifically to Round 10 RNA (Fig. 7A). DNA sequence analysis of the RING finger domain of the PCR clone and the $\mathrm{P}_{2}$ clone revealed a single point mutation in the PCR clone which produced a glycine-to-serine change at amino acid 446 (GGC to AGC) (Fig. 7B). This mutant MDM2 protein failed to bind to Round 10 RNA.

\section{DISCUSSION}

The MDM2 protein was initially tested to determine if it was capable of binding to RNA because of the presence of the central zinc finger and C-terminal RING finger domains in the protein, the ability of the protein to bind to a heparinsepharose column, and the association of MDM2 with the L5 protein/5S rRNA ribonucleoprotein. An RNA homopolymer binding assay was used as an initial assay to test whether this protein had the ability to bind RNA as was the case for several other RNA-binding proteins (29-31). The specificity of MDM2 for $\operatorname{poly}(\mathrm{G})$ and its binding in relatively high salt concentrations is very similar to that of the FMRI RNA-binding protein (26). A mutation in the FMRl gene causes fragile $\mathrm{X}$ syndrome. The pattern of poly $(G)$ binding is also similar to that of the yeast protein Nupl45p, a nucleoporin involved in nucleocytoplasmic transport (32). The possibility that the poly $(G)$ RNA binding by MDM2 was mediated through the L5 protein was eliminated by mapping the regions on MDM2 responsible for binding to the L5 protein and poly $(G)$ RNA. These data demonstrate that mutations in the central region which 
FIG. 7. A point mutation in the RING finger domain of MDM2 abolishes specific RNA binding

(A) MDM2 expressed from either the PCR clone, which contains the point mutation, or the $\mathrm{P}_{2}$ clone were tested for RNA-binding activity with the Round 10 RNA. Immunoprecipitations were performed from the indicated Sf9 cell lysates with the 419 negative control $\mathrm{mAb}$ or the $4 \mathrm{~B} 2$ and 2A10 MDM2 mAbs. (B) Amino acid sequence comparison of the C-terminal 68 amino acids of HDM 2 and MDM2. Identical amino acids are indicated by an asterix and conservative amino acid differences by a period. Numbers indicate amino acid positions of HDM2 and MDM2. A G446S mutation in MDM2 abolishes its ability to bind specifically to Round 10 RNA.
A.

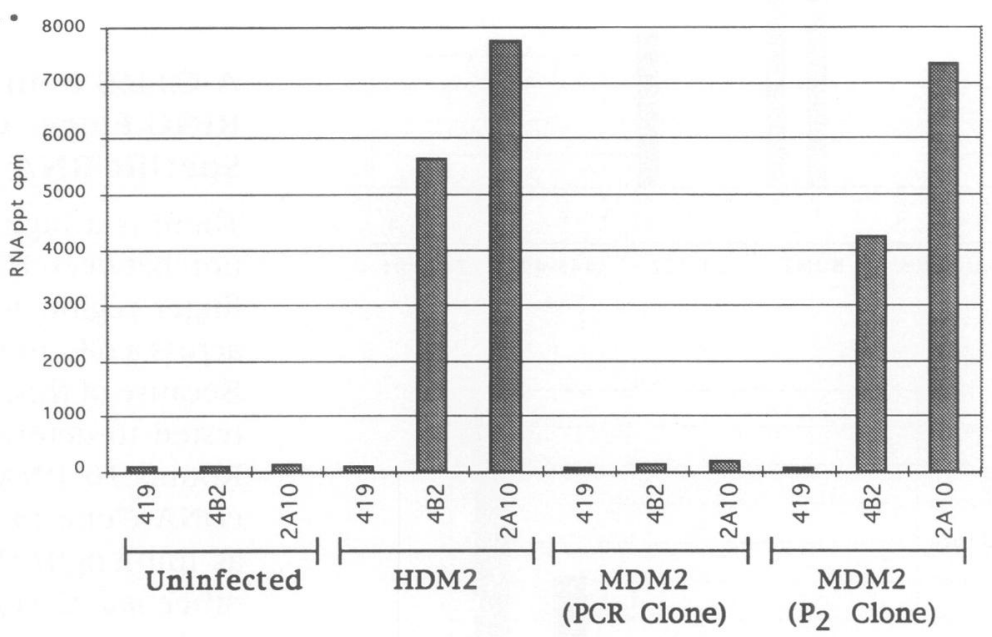

B.

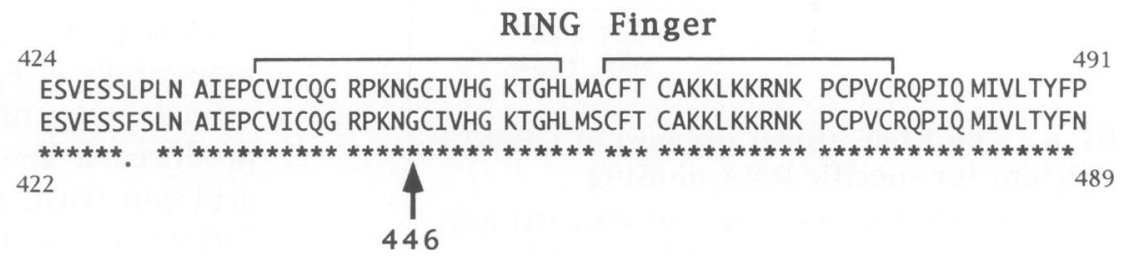

eliminate L5 protein binding retain the ability to bind poly(G) RNA.

Next, a SELEX procedure was employed to determine whether the MDM2 protein was capable of binding specific RNA sequences or structures and, if so, to identify a pool of such specific RNA molecules. The SELEX procedure has been used successfully to identify high-affinity RNA ligands for a variety of proteins $(27,28,36)$. The pool of high-affinity RNA molecules that bound to HDM2 (Round 10) binds entirely to the RING finger domain of the protein. This domain is very well conserved during evolution (V. Marechal et al., in preparation) and therefore likely has an important biological function. The data presented suggest that this function may involve interaction with RNA. In this context, it is of interest that a single point mutation in this domain, G446S, completely abolishes the specific RNA binding. This $m d m 2$ mutant will be useful in identifying possible functions for this activity.

One possible function for the specific RNA binding of the RING finger is that it interacts with the $5 \mathrm{~S}$ rRNA since MDM2 is known to form a complex with the L5 protein and $5 \mathrm{~S}$ rRNA ribo- nucleoprotein. This is unlikely for several reasons. First, RNA sequences similar to those isolated from the SELEX procedure are not seen in the $5 \mathrm{~S}$ rRNA. Second, MDM2 produced from the PCR clone containing the point mutation binds as efficiently to L5 as wild-type MDM2 or HDM2. Lastly, when labeled 5S rRNA was incubated with MDM2 protein in the RNA-binding assay, no binding was detected (data not shown).

Previously published results suggest that the p53 tumor suppressor protein can be found as part of a multimeric complex consisting of MDM2, L5, 5S rRNA, and possibly 5.8S rRNA (18). Since p 53 has the ability to interact with RNA $(37,38)$, whether p53 could contribute to the RNA-binding specificity of a complex containing both MDM2 and p53 was of interest. To determine whether the RNA-binding activity of p53 was specific for certain RNA species, a SELEX experiment was performed with $\mathrm{p} 53$ as a target. After 10 rounds of selection and amplification, the overall affinity of the pools had increased only slightly (one order of magnitude) when compared with the initial SELEX library. The final pool of RNA ligands did not display any 
recognizable common feature with respect to sequence or structure (M. Dobbelstein, unpublished observations). We therefore assume that p53 may contribute to the RNA-binding activity of a complex containing both p53 and MDM2, but probably does not significantly affect its specificity for certain RNA motifs.

While the RING finger is sufficient for the specific binding to small structured RNAs isolated from the SELEX procedure, HDM2 may have a second RNA-binding region since the HDM2 deletion mutant lacking the RING finger retains poly $(G)$ binding activity. The second domain maps to the central region as shown by the fact that the $\Delta 295-491$ mutant binds poly $(G)$ but not the $\Delta 205-491$ mutant. However, it cannot be ruled out that, in this case, HDM2 may bind indirectly to poly $(\mathrm{G})$ through traces of insect cell L5 protein, a known RNA-binding protein, that could be present in the HDM2 preparation.

The RING finger domain is a newly characterized $\mathrm{C}_{3} \mathrm{HC}_{4}$ zinc-binding domain initially identified in the human ringl gene (23). The tertiary structures of two RING finger domains from the IE 110 protein of equine herpes simplex virus and the PML proto-oncoprotein have been solved in solution by ${ }^{1} \mathrm{H}-\mathrm{NMR}(21,22)$. In both structures, four pairs of metal binding residues coordinate two molecules of zinc, with the first and third pairs binding one molecule of zinc and the second and fourth pairs binding a second. Interestingly, the IE1 10 protein adopts a $\beta \beta \alpha \beta$ fold, and the region comprising the first two $\beta$ strands and the $\alpha$-helix has remarkable similarity with a TFIIIA type of zinc finger (21). Xenopus TFIIIA has nine zinc fingers which are involved in sequence-specific DNA binding to the $5 \mathrm{~S}$ ribosomal gene and specific RNA binding to the $5 \mathrm{~S}$ rRNA $(39,40)$. This similarity is in agreement with the finding described here that a RING finger domain can mediate interaction with RNA.

RING finger domains have been found in a growing number of proteins in a variety of species and they have a wide range of functions (24). Examples of RING finger proteins include the BRCAl tumor suppressor protein (41), the PML proto-oncoprotein (42), the baculovirus inhibitor of apoptosis IAP (43), Msl-2 which is required for $\mathrm{X}$ chromosome dosage compensation in Drosophila males (44), and the yeast RAD5 protein involved in DNA repair (45). In some cases, such as Msl-2 and RAD5, the RING finger has been proposed to play a role in protein-DNA interaction, but so far no RING finger protein has been shown to possess sequence- specific DNA-binding activity. Although the function of this domain is still unclear, the RING finger may constitute a multifunctional domain involved in either protein-protein interaction or protein-nucleic acid interaction $(22,24)$. Based upon the experiments reported here, the RING finger of MDM2 is clearly capable of binding specifically to RNA. This may define the RING finger as a novel RNA-binding motif (46).

Both the L5 binding and specific RNA-binding functions are conserved between mouse and human forms of MDM2. However, it is not known how these functions may contribute to the oncogenic potential of the protein. The complex formation with the L5/5S ribosomal ribonucleoprotein and the ability to bind specifically to RNA present the intriguing possibility that MDM2 may be localized at the ribosome and function in regulating the translation of specific mRNAs involved in the control of cell growth. Further experiments are needed to address the possibility that MDM2 is localized to the ribosome and can affect gene expression or translation as well as the contribution of this activity to its ability to regulate cell growth.

\section{ACKNOWLEDGMENTS}

We are grateful to Jiandong Chen for providing the HDM2 deletion mutant expression plasmids and for helpful advice. We thank Maureen Murphy for her critical reading of this manuscript and Trisha Barney for help in preparing the manuscript. This work was supported by a grant from the National Cancer Institute to A. J. Levine and T. Shenk (CA41086). B. Elenbaas was supported by a fellowship from the New Jersey Commission on Cancer Research. M. Dobbelstein was supported by the Stipendium fur Infektionsbiologie of the German Cancer Research Center. J. Roth was supported by a fellowship of the Deutsche Forschungsgemeinschaft. T. Shenk is an American Cancer Society professor and an Investigator of the Howard Hughes Medical Institute.

\section{REFERENCES}

1. Fakharzadeh SS, Trusko SP, George DL. (1991) Tumorigenic potential associated with enhanced expression of a gene that is amplified in a mouse tumor cell line. $E M B O$ J. 10: 1565-1569. 
2. Finlay CA. (1993) The mdm-2 oncogene can overcome wild-type p53 suppression of transformed cell growth. Mol. Cell. Biol. 13: 301-306.

3. Cordon-Cardo C, Latres E, Drobnjak M, et al. (1994) Molecular abnormalities of $\mathrm{mdm} 2$ and $\mathrm{p} 53$ genes in adult soft tissue sarcomas. Cancer Res. 54: 794-799.

4. Ladanyi $M$, Cha C, Lewis R, Jhanwar SC, Huvos AG, Healy JH. (1993) Mdm-2 gene amplification in metastatic osteosarcoma. Cancer Res. 53: 16-18.

5. Leach FS, Tokino T, Meltzer P, et al. (1993) p53 mutation and mdm2 amplification in human soft tissue sarcomas. Cancer Res. 53: 2231-2234.

6. Oliner JD, Kinzler KW, Meltzer PS, George DL, Vogelstein B. (1992) Amplification of a gene encoding a $\mathrm{p} 53$-associated protein in human sarcomas. Nature 358: 80-83.

7. Reifenberger G, Liu L, Ichimura K, Schmidt EE, Collins VP. (1993) Amplification and overexpression of the $\mathrm{mdm} 2$ gene in a subset of human malignant gliomas without p53 mutations. Cancer Res. 53: 2736-2739.

8. Berberich S, Cole M. (1994) The mdm-2 oncogene is translocated and overexpressed in a murine plasmacytoma cell line expressing wild-type p53. Oncogene 9: 1469-1472.

9. Bueso-Ramos CE, Yang $\mathrm{Y}$, deLeon E, McCown P, Stass SA, Albitar M. (1993) The human mdm-2 oncogene is overexpressed in leukemias. Blood 82: 2617-2623.

10. Landers JE, Haines DS, Strauss JF, George DL. (1994) Enhanced translation: A novel mechanism of mdm2 overexpression identified in human tumor cells. Oncogene 9: 27452750.

11. Chen J, Marechal V, Levine AJ. (1993) Mapping of the p53 and mdm-2 interaction domains. Mol. Cell. Biol. 13: 4107-4114.

12. Momand J, Zambetti GP, Olson DC, George D, Levine AJ. (1992) The mdm-2 oncogene product forms a complex with the $\mathrm{p} 53$ protein and inhibits p53-mediated transactivation. Cell 69: 1237-1240.

13. Oliner JD, Pietenpol JA, Thiagalingam S, Gyuris J, Kinzler KW, Vogelstein B. (1993) Oncoprotein MDM2 conceals the activation domain of tumor suppressor p53. Nature 362: $857-860$.

14. Jones SN, Roe AE, Donehower LA, Bradley A. (1995) Rescue of embryonic lethality in Mdm2-deficient mice by absence of p53. $\mathrm{Na}$ ture 378: 206-208.
15. Montes de Oca Luna R, Wagner DS, Lozano G. (1995) Rescue of early embyronic lethality in mdm2-deficient mice by deletion of p53. Nature 378: 203-206.

16. Xiao Z, Chen J, Levine AJ, et al. (1995) Interaction between the retinoblastoma protein and the oncoprotein MDM2. Nature 375: 694-698.

17. Martin K, Trouche D, Hagemeier C, Sorensen S, La Thangue NB, Kouzarides $\mathrm{T}$. (1995) Stimulation of E2Fl/DPl transcriptional activity by MDM2 oncoprotein. Nature 375: 691-694.

18. Marechal V, Elenbaas B, Piette J, Nicolas J, Levine AJ. (1994) The ribosomal L5 protein is associated with mdm-2 and mdm-2-p53 complexes. Mol. Cell. Biol. 14: 7414-7420.

19. Chen J, Lin J, Levine AJ. (1995) The regulation of p53-mediated transcriptional functions by mdm-2. Mol. Med. 1: 142-152.

20. Boddy MN, Freemont PS. (1994) The p53associated protein MDM2 contains a newly characterized zinc-binding domain called the RING finger. TIBS 19: 198-199.

21. Barlow PN, Luisi B, Milner A, Elliott $M$, Everett R. (1994) Structure of the $\mathrm{C}_{3} \mathrm{HC}_{4}$ domain by ${ }^{1} \mathrm{H}$-nuclear magnetic resonance spectroscopy. J. Mol. Biol. 237: 201-211.

22. Borden KLB, Boddy MN, Lally $\mathrm{J}$, et al. (1995) The solution structure of the RING finger domain from the acute promyelocytic leukaemia proto-oncoprotein PML. EMBO J. 14: 1532-1541.

23. Lovering $R$, Hanson $M$, Borden KLB, et al. (1993) Identification and preliminary characterization of a protein motif related to the zinc finger. Proc. Natl. Acad. Sci. U.S.A. 90: 2112-2116.

24. Freemont PS. (1993) The RING finger. A novel protein sequence motif related to the zinc finger. Ann. N.Y. Acad. Sci. 684: 174192.

25. Harlow E, Lane D. (1988) Antibodies: A Laboratory Manual. Cold Spring Harbor Press, Cold Spring Harbor, NY.

26. Siomi H, Siomi MC, Nussbaum RL, Dreyfuss G. (1993) The protein product of the fragile $X$ gene, FMRl, has characteristics of an RNA-binding protein. Cell 74: 291-298.

27. Dobbelstein M, Shenk T. (1995) In vitro selection of RNA ligands for the ribosomal L22 protein associated with Epstein-Barr virusexpressed RNA by using randomized and cDNA-derived RNA libraries. J. Virol. 69: 8027-8034. 
28. Schneider D, Gold L, Platt T. (1993) Selective enrichment of RNA species for tight binding to Escherichia coli rho factor. FASEB J. 7: 201207.

29. Dreyfuss G, Matunis MJ, Pinol-Roma S, Burd CG. (1993) hnRNP proteins and the biogenesis of mRNA. Annu. Rev. Biochem. 62: 289-321.

30. Kiledjian M, Dreyfuss G. (1992) Primary structure and binding activity of the hnRNP U protein: Binding RNA through RGG box. EMBO J. 1: 2655-2664.

31. Swanson MS, Dreyfuss G. (1988) Classification and purification of proteins of heterogeneous nuclear ribonucleoprotein particles by RNA-binding proteins. Mol. Cell. Biol. 3: 2237-2241.

32. Fabre E, Boelens WC, Wimmer C, Mattaj IW, Hurt EC. (1994) Nupl45p is required for nuclear export of mRNA and binds homopolymeric RNA in vitro via a novel conserved motif. Cell 78: 275-289.

33. Zuker M. (1994) Prediction of RNA secondary structure by energy minimization. Methods Mol. Biol. 25: 267-294.

34. Tian Y, Adya N, Wagner S, Giam C, Green MR, Ellington AD. (1995). Dissecting protein:protein interactions between transcription factors with an RNA aptamer. RNA 1: 317-326.

35. Barak Y, Gottlieb E, Juven-Gershon T, Oren M. (1994) Regulation of $m d m 2$ expression by p53: Alternative promoters produce transcripts with nonidentical translation potential. Genes Dev. 8: 1739-1749.

36. Tuerk C, MacDougal S, Gold L. (1992) RNA pseudoknots that inhibit human immunodeficiency virus type 1 reverse transcriptase. Proc. Natl. Acad. Sci. U.S.A. 89: 6988-6992.

37. Oberosler $P$, Hloch $P$, Ramsperger U, Stahl H. (1993) p53-catalyzed annealing of complementary single-stranded nucleic acids. EMBO J. 12: 2389-2396.

Contributed by A. J. Levine on May 14, 1996.
38. Wu L, Bayle JH, Elenbaas B, Pavletich NP, Levine AJ. (1995) Alternatively spliced forms in the carboxy-terminal domain of the p53 protein promote annealing of complementary single strands of nucleic acids. Mol. Cell. Biol. 15: 497-504.

39. Sands MS, Bogenhagen DF. (1991) The carboxy-terminal zinc fingers of TFIIIA interact with the tip of helix V of 5S RNA in the 7S ribonucleoprotein particle. Nucleic Acids Res. 19: 1791-1796.

40. Theunissen $\mathrm{O}$, Rudt $\mathrm{F}$, Guddat $\mathrm{U}$, Mentzel $\mathrm{H}$, Pieler T. (1992) RNA and DNA binding zinc fingers in Xenopus TFIIIA. Cell 71: 679-690.

41. Miki Y, Swensen J, Shattuck-Eidens D, et al. (1994) A strong candidate for the breast and ovarian cancer susceptibility gene BRCA1. Science 266: 66-71.

42. Goddard AD, Borrow J, Freemont PS, Solomon E. (1991) Characterization of a zincfinger gene disrupted by the $t(15 ; 17)$ in acute promyelocytic leukemia. Science 254: 1371-1374.

43. Crook NE, Clem RJ, Miller LK. (1993) An apoptosis-inhibiting baculovirus gene with a zinc finger-like motif. J. Virol. 67: 21682174.

44. Kelley RL, Solovyeva I, Lyman M, Richman R, Solovyev V, Kuroda MI. (1995) Expression of Msl-2 causes assembly of dosage compensation regulators on the $\mathrm{X}$ chromosomes and female lethality in Drosophilia. Cell 81: 867-877.

45. Johnson RE, Henderson ST, Petes TD, Prakash S, Bankmann M, Pakash L. (1992) Saccharomyces cerevisiae RAD5-encoded DNA repair protein contains DNA helicase and zinc-binding sequence motifs and affects the stability of simple repetitive sequences in the genome. Mol. Cell. Biol. 12: 3807-3818.

46. Burd CG, Dreyfuss G. (1994) Conserved structures and diversity of functions of RNAbinding proteins. Science 265: 615-621. 